\title{
Improvement of DWT-SVD with Curve Fitting and Robust Regression: An Application to Astronomy Images
}

\author{
Talha Karadeniz ${ }^{1}$, Ersin Elbasi ${ }^{2}$ \\ ${ }^{I}$ Department of Computer Engineering, Cankaya University, \\ Ankara, Turkey \\ ${ }^{2}$ Computer and Information Science, Higher Colleges of Technology \\ Al Ain, UAE \\ eelbasi@hct.ac.ae
}

\begin{abstract}
DWT-SVD is a frequency domain based eigenanalysis watermarking technique. In this work, we improve this method by exploring the relationship between the cover image's DWT singular values and those of the watermark. We show that, via the usage of curve fitting and robust regression, it is possible to achieve accurate results. We also demonstrate that the improved scheme is suitable for the watermarking of astronomy images. In addition to encoding and decoding examples, statistical results on stealth and robustness are deduced from the experiments so that the clear advance can be observed. Quality of the watermark is measured by testing against various attack types.
\end{abstract}

Index Terms-Watermarking; discrete wavelet transforms; eigenvalues and eigenfunctions; curve fitting.

\section{INTRODUCTION}

Digital watermarking has a wide area of application. The most important one of these is copyright security; a secret message is embedded into the media so that the owner knows it stands as a signature. For the battle against piracy and for the management of media tracking, it is impossible to neglect the benefit of information hiding [1]-[5].

The domain of visual watermarking is classified into two types. The first one is the obvious one where the pixels reside; spatial domain. The second one is the frequency domain, where we embed the secret message into the frequency coefficients obtained via an analysis of the cover image. Discrete Wavelet Transform (DWT) and Discrete Cosine Transform (DCT) are examples of the state-of-theart frequency domain analysis. In basic DWT embedding, the visual watermark is hidden into the wavelet coefficients of the cover image [6]-[10]. That is, coefficients are modified by using the intensity values of the watermark. In DWT-SVD embedding, singular values of the frequency coefficients are modified by using the singular values of the visual watermark. In this work, we focus on the improvement of DWT-SVD technique [11].

Although the central technique DWT is somehow old, its modified and upgraded versions, that is, strengthened ways of frequency domain analysis via SVD or other

Manuscript received 8 August, 2015; accepted 19 January, 2016. transformations, are still in the field of active research [12], [13]. Moreover, even DWT itself is engineered in more articulated techniques [14], which is a proof of the solid state of this classical algorithm. Variants such as DCT are also up-to-date base selections for implementations of watermarking [15].

Influenced by [10], we have derived a technique which will be explained as follows: in II.A base theoretical tools, in II.B and II.C extensions such as curve fitting are noted. Overall scheme development and experiment details are given in II.D, II.E, respectively.

\section{IMPROVEMENT OF DWT-SVD}

Our aim at this work was to establish an improvement of DWT-SVD [10], [11], [14] via an additional analysis on singular values. For this, we utilized curve fitting and robust regression. Thus, the derived technique can be summarized as DWT-SVD-CF-RR (DSCR). The ideal advance of a watermarking scheme must be in both robustness and stealth. Robustness is the 'strength' measure of the encoding to a range of attacks. Stealth, on the other hand, is the transparency of the hidden message; this is essential for the overall commercial quality of the modified image.

Before carrying out the experiments, our main idea was this: what if one embeds an approximation of watermark singular values as a function of the cover DWT singular values rather than directly using the initial (original) ones? This corresponds to a more consistent modification since the same function is used for the band's all singular values; the resulting sequence is retrieved by a well-defined single variable function. On the other hand, since an approximation is used, the connection to the watermark is almost retained. Hence, our initial guess was that the final PSNR value of the encoded image should be higher than that of the standard DWT-SVD's output and the correlation values of the decoded singular values should have a tolerable distance to those of the standard strategy. After experiments, we have seen that, both PSNR values and correlation measurements were better.

\section{A. DWT-SVD}

Assume that we have a $2 \mathrm{n} \times 2 \mathrm{n}$ cover and an $\mathrm{n} \times \mathrm{n}$ 
watermark image.

\section{1) Embedding}

1. Apply DWT and get subbands $L L, L H, H L$ and $H H$.

2. Apply SVD to the watermark image and get $\mathbf{U}_{\mathbf{w}} \boldsymbol{\Sigma}_{\mathbf{w}} \mathbf{V}_{\mathbf{w}}^{\mathbf{T}} \cdot \boldsymbol{\Sigma}_{\mathbf{w}}$ is a diagonal matrix containing the singular values $\lambda_{w_{1}}, \lambda_{w_{2}}, \ldots, \lambda_{w_{n}} . \mathbf{U}_{\mathbf{w}}$ and $\mathbf{V}_{\mathbf{w}}$ are orthogonal matrices.

3. For i-th sub band apply SVD and get the decomposition $\mathbf{U}_{\mathbf{i}} \boldsymbol{\Sigma}_{\mathbf{i}} \mathbf{V}_{\mathbf{i}}^{\mathbf{T}}$. Make this for all $\mathbf{i}=1,2,3,4$, where $\mathrm{i}=1,2,3,4$ corresponds to indices of $\mathrm{LL}, \mathrm{LH}, \mathrm{HL}$ and $\mathrm{HH}$ respectively.

4. Modify the subbands as $\mathbf{U}_{\mathbf{i}}\left(\boldsymbol{\Sigma}_{\mathbf{i}}+\alpha_{i} \boldsymbol{\Sigma}_{\mathbf{w}}\right) \mathbf{V}_{\mathbf{i}}^{\mathbf{T}}$, where $\alpha_{i}$ is a scaling factor. That is, modify the singular values of $i-$ th subband as $\lambda_{i_{1}}+\alpha_{i} \lambda_{w_{1}}, \lambda_{i_{2}}+\alpha_{i} \lambda_{w_{2}}, \ldots, \lambda_{i_{n}}+\alpha_{i} \lambda_{w_{n}}$ and apply inverse SVD transform.

5. Apply inverse transform to the subbands to get the watermarked image.

\section{2) Extraction}

1. Apply DWT to the input image $I^{*}$ and get subbands LL, LH, HL and HH.

2. For i-th subband apply SVD and get the decomposition $\mathbf{U}_{\boldsymbol{i}}^{*} \Sigma_{\boldsymbol{i}}^{*} \boldsymbol{V}_{\boldsymbol{i}}^{*} \boldsymbol{T}$. Make this for all $\mathrm{i}=1,2,3,4$, where $\mathrm{i}=1$, 2, 3, 4 corresponds to LL, LH, HL and HH respectively.

3. Find the constructed singular values $\left(\lambda^{*}{ }_{i_{j}}-\lambda_{w_{j}}\right) / \alpha_{i}$ for $\mathrm{j}=1,2, \ldots, \mathrm{n}$.

4. Output the visual watermark as $\mathbf{U}_{\mathbf{w}} \Sigma_{w}^{c} \mathbf{V}_{\mathbf{w}}^{\mathbf{T}}$ where $\Sigma_{w}^{c}$ is a diagonal matrix containing the values $\left(\lambda^{*}{ }_{i_{j}}-\lambda_{w_{j}}\right) / \alpha_{i}$ for $\mathrm{j}=1,2, \ldots, \mathrm{n}$.

\section{B. Curve Fitting}

Assume that we have $\mathrm{m}$ points $\left\{\left(x_{1}, y_{1}\right),\left(x_{2}, y_{2}\right), \ldots,\left(x_{m}, y_{m}\right)\right\}$. What we do at (leastsquares) curve fitting is to find a $d$ degree polynomial

$$
f_{1}(x)=a_{d} x^{d}+a_{d-1} x^{d-1}+\ldots+a_{1} x+a_{0}
$$

such that $\sum_{i=1}^{m}\left(f_{1}\left(x_{i}\right)-y_{i}\right)^{2}$ is minimized. We formulated curve fitting for the LL band as follows: for a given degree $d$, find $f_{1}(x)$ such that $\sum_{i=1}^{n}\left(f_{1}\left(\lambda_{1_{i}}\right)-\lambda_{w_{i}}\right)^{2}$ is minimized. Hence, we fit a polynomial of LL band singular values to approximate the singular values of the watermark.

\section{Robust Linear Regression}

Ordinary Least Squares (OLS) is formulized as follows: given $\boldsymbol{X}, \boldsymbol{y}$ matrices where the dimensions are $\mathrm{n} \times \mathrm{D}$ and $\mathrm{n} \times 1$ respectively, find $\mathrm{D} \times 1$ dimensional matrix $\boldsymbol{\beta}=\hat{\boldsymbol{\beta}}$ such that

$$
\boldsymbol{X \beta}-\boldsymbol{y}
$$

is minimized. That is, we model the relationship between an input vector $\mathbf{x}$ and a target value $\mathbf{y}$ in the form of a linear function characterized by $\boldsymbol{\beta}$, where the prediction $\hat{y}$ is evaluated as

$$
\widehat{y}=x_{1} \beta_{1}+x_{2} \beta_{2}+\ldots+x_{D} \beta_{D},
$$

so that the total distance of the estimated values to the actual ones are minimized.

While modeling via OLS, we do not take the weights of the samples into account. If we do it, the result is a new formulation which contains the analysis of outliers and leverage points; Weighted Least Squares (WLS). Outliers are points that are not fitted very well by the linear model (i.e. points having large residuals), whereas leverage points are those outlying in the $x$-space [3]. Since the weight assigning procedure is dependent to residuals and residuals are calculated at each iteration, weighting is done iteratively. Robust Regression can be summarized as solving

$$
\operatorname{argmin}_{\boldsymbol{\beta}} \sum_{i=1}^{n} w_{i}^{j}\left(y_{i}-x_{i_{1}} \beta_{1}+x_{i_{2}} \beta_{2}+\ldots+x_{i_{D}} \beta_{D}\right),
$$

at each $\mathrm{j}$-th iteration. Weighting is done according to the residuals of the last fit

$$
\boldsymbol{r}^{\boldsymbol{j}-1}=\boldsymbol{y}-\boldsymbol{y}^{\boldsymbol{j}-1}=\boldsymbol{y}-\boldsymbol{X} \hat{\boldsymbol{\beta}}^{\boldsymbol{j}-1}
$$

and the leverage matrix

$$
H=X\left(X^{T} X\right)^{-1} X^{T}
$$

\section{Scheme}

Assume that we have a $2 n \times 2 n$ cover and an $n \times n$ watermark image.

1) Embedding

Given $\left\{\alpha_{1}, \alpha_{2}, \alpha_{3}, \alpha_{4}, t\right\} \in \mathbb{R}, \quad \mathrm{s}={ }^{\prime} s_{1} s_{2} s_{3} s_{4} ", \quad d \in \mathbb{N}$ where $\alpha_{i}$ are the scaling factors of DWT- SVD, $\mathrm{t}$ is the tuning constant of robust regression, $\mathrm{s}$ is a binary string (i.e. $s_{i} \in\left\{0^{\prime}, 1^{\prime}\right\}$ and $d>1$ is the degree of the curve fitting polynomial.

1. Apply DWT and get subbands $L L, L H, H L$ and $H H$.

2. Apply SVD to the watermark image and get $\mathbf{U}_{\mathbf{w}} \boldsymbol{\Sigma}_{\mathbf{w}} \mathbf{V}_{\mathbf{w}}^{\mathbf{T}} \cdot \boldsymbol{\Sigma}_{\mathbf{w}}$ is a diagonal matrix containing the singular values $\lambda_{w_{1}}, \lambda_{w_{2}}, \ldots, \lambda_{w_{n}}, \mathbf{U}_{\mathbf{w}}$ and $\mathbf{V}_{\mathbf{w}}$ are orthogonal matrices.

3. For i-th subband apply SVD and get the decomposition $\mathbf{U}_{\mathbf{i}} \boldsymbol{\Sigma}_{\mathbf{i}} \mathbf{V}_{\mathbf{i}}^{\mathbf{T}}$. Make this for all $\mathrm{i}=1,2,3,4$, where $\mathrm{i}=1,2$, 3,4 corresponds to indices of LL, LH, HL and $\mathrm{HH}$ respectively.

4. Find the polynomial fitting function $\mathrm{f}_{1}$ of degree $\mathrm{d}$, where the input set is $\left\{\left(\lambda_{1_{1}}, \lambda_{w_{1}}\right),\left(\lambda_{1_{2}}, \lambda_{w_{2}}\right), \ldots,\left(\lambda_{1_{n}}, \lambda_{w_{n}}\right)\right\}$, i.e. fit a function of singular values of the LL band with the output values of the watermark's singular values.

5. For $i \in\{2,3,4\}$ if $s_{i}=' 1$ ', use robust regression to find

a least-squares approximation $f_{i}$ on 
$\left\{\left(\lambda_{i_{1}}, \lambda_{w_{1}}\right),\left(\lambda_{i_{2}}, \lambda_{w_{2}}\right), \ldots,\left(\lambda_{i_{n}}, \lambda_{w_{n}}\right)\right\}$. i.e. if $s_{2}={ }^{\prime} 1 '$ utilize robust regression on the singular values $\lambda_{2}$ of the LH band to find $f_{2}\left(\lambda_{2}\right) \approx \lambda_{w_{j}}$. Similarly, if $s_{3}=$ '1', utilize robust regression on the singular values $\lambda_{3_{j}}$ of the HL band to find $f_{3}\left(\lambda_{3_{j}}\right) \approx \lambda_{w_{j}}$. If $s_{4}=' 1$ ', follow the same route for the $\mathrm{HH}$ band.

6. Fit a fusion model $F$ based on multi-dimensional robust regression to exploit the relationship between input vectors $\left(f_{1}\left(\lambda_{1_{j}}\right), f_{2}\left(\lambda_{2}\right), f_{3}\left(\lambda_{3_{j}}\right), f_{4}\left(\lambda_{4_{j}}\right)\right)$ and the output values $\lambda_{w_{j}}$ Of course, this is for the case $s=" 1111 "$. If, for instance, $s=" 1011 "$, we would use $\left(f_{1}\left(\lambda_{1_{j}}\right), f_{3}\left(\lambda_{3_{j}}\right), f_{4}\left(\lambda_{4_{j}}\right)\right)$.

7. If $s_{i}={ }^{\prime} 0^{\prime}$, modify the corresponding subband as $\mathbf{U}_{\mathbf{i}}\left(\boldsymbol{\Sigma}_{\mathbf{i}}+\alpha_{\mathbf{i}} \boldsymbol{\Sigma}_{\mathbf{w}}\right) \mathbf{V}_{\mathbf{i}}^{\mathbf{T}}$, (as in II.A.1). Otherwise modify it as $\mathbf{U}_{\mathbf{i}}\left(\boldsymbol{\Sigma}_{\mathbf{i}}+\alpha_{\mathrm{i}} \boldsymbol{\Sigma}_{\boldsymbol{w}}^{f_{i}}\right) \mathbf{V}_{\mathbf{i}}^{\mathbf{T}}$ where $\boldsymbol{\Sigma}_{\boldsymbol{w}}^{f_{i}}$ stands for the the diagonal matrix consisting the singular value approximations $\left\{f_{I}\left(\lambda_{i_{1}}\right), f_{I}\left(\lambda_{i_{2}}\right), \ldots, f_{i}\left(\lambda_{i_{n}}\right)\right\}$.

8. Apply inverse DWT transform to the subbands to get the watermarked image.

\section{2) Extraction}

Extraction is almost the same as in II.A.2. An additional step of fusion via $F$ (II.D.1) is added. Say, we have $s=$ "1111". (i.e. we utilize robust regression for all LH, HL, $\mathrm{HH}$ bands). If reconstructed singular values are $\left(\lambda_{1_{j}}^{c}, \lambda_{2_{j}}^{c}, \lambda_{3_{j}}^{c}, \lambda_{4_{j}}^{c}\right)$ then we use $F$ to find $\lambda_{j}^{F}$ (for $\mathrm{j}=1,2$, ..., n). This means that, we have an alternative fusion sequence of singular values $\left\{\lambda_{1}^{F}, \lambda_{2}^{F}, \ldots, \lambda_{n}^{F}\right\}$.

\section{3) Summary}

Given the details of the embedding and extraction routines, the overall algorithm can be summarized as follows:

1. Given the input and watermark images, calculate DWT transformations.

2. For input and watermark images:

a. Calculate SVD of each subband.

3. Express the input singular values in terms of the watermark singular values via a curve fitting or a robust regression analysis.

4. Follow the regular DWT-SVD steps except the direct embedding of the cover eigenvalues.

5. Strengthen the stealth and the robustness by switching the on-off status of subband eigenvalue modification.

\section{E. Experiments}

\section{1) Implementation}

All code for experiments are written in MATLAB. Given an image and DWT scaling factors $\left\{\alpha_{1}, \alpha_{2}, \alpha_{3}, \alpha_{4}\right\}$, we found optimal $d, t$ and $s$. Optimality is calculated in terms of PSNR (stealth) and mean Pearson correlation value (robustness) under a range of attacks. We started with $s=" 1111 "$, tried several values for $t$ and $d$, only if an improvement - a greater PSNR of the encoded image and a higher mean correlation value - couldn't be found, we modified $s$ gradually; we first tried $s=" 1110 "$, later $s=" 1101 "$. and so on. Here, $s$ is of length 4 for the sake of convenience; we never omitted curve fitting approximation embedded in LL band - modification. Choosing the high- PSNR values of the original DWT-SVD algorithm, we set $\alpha_{1}=0.05, \alpha_{2}=\alpha_{3}=\alpha_{4}=0.005$. We used bisquare weighting and the same $t$ for all robust regression steps.

Reported mean correlation and PSNR values are obtained when DSCR got a better result compared to DWT-SVD, i.e. these are not the best values, albeit the superior outcome.

2) Astronomy Images

This work started with an application-driven idea of implementing a visual watermark algorithm to the domain of astrophotography. We used Hubble Site [4] for the dataset of images.

Examples of deep sky images are shown in Fig. 1.

- Messier 101: a spiral galaxy (Fig. 1(a)). Estimated number of stars it consists is about one trillion.

- NGC 290: a star cluster in the Small Magellanic Cloud (Fig. 1(b)).

- Messier 74: also known as NGC 628, a spiral galaxy slightly smaller than Milky Way (Fig. 1(c)).

- LH 95: a star forming region of glowing hydrogen in the Large Magellanic Cloud [3] (Fig. 1(d)).

Using these images and performing a sequence of 10 attacks: JPEG 75 (JPEG compression with quality 75), JPEG 50 (JPEG compression with quality 50), JPEG 25 (JPEG compression with quality 25), Gaussian Noise (with 0 mean and 0.001 variance), Mean Filter (2-D filter mean filter), Resize $(512 \times 512 \rightarrow 256 \times 256 \rightarrow 512 \times 512$ bilinear resizing), Rotation (20 degrees), Histogram Equalization (Contrast enhancement via histogram equalization), Intensity Adjustment (Intensity interval [0, 0.8] mapped to $[0,1]$ ), Gamma Correction (Intensity adjustment with gamma $=1$ ).

On the encoded image, we measured PSNR and mean correlation. Each correlation value is recorded as in [9], i.e. by taking the highest of all bands (including the fusion result 2.4.1). Differing from [1], we used mean correlation as a final robustness metric

$$
\frac{1}{N} \sum_{i=1}^{N} \operatorname{corr}\left(\lambda_{i}, \lambda_{w}\right)
$$

where $\lambda_{i}$ and $\lambda_{w}$ are the $\mathrm{i}$-th reconstructed singular value vector (from the $i$-th attacked image) and the visual watermark's singular value vector, respectively. corr is Pearson correlation function.

For NGC 290, the original, encoded and decoded images can be seen in Fig. 2. Detailed results for M101, NGC 290, M74 and LH 95 can be seen in Table I. 


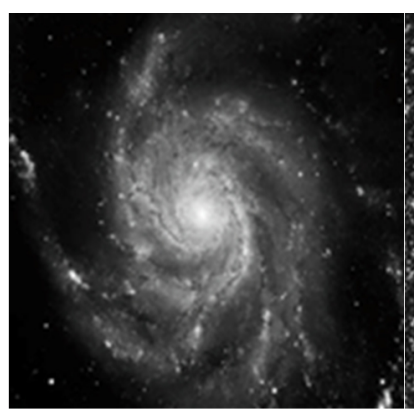

(a)

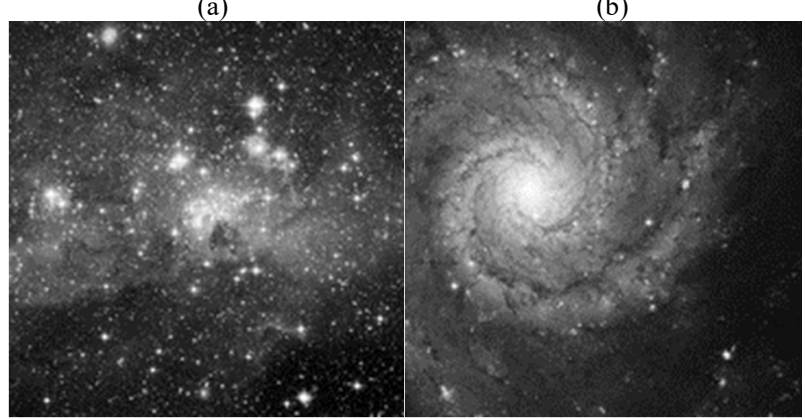

(c)

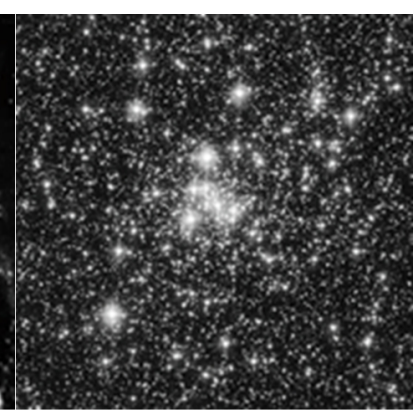

(b)

(d)
Fig. 1. Deep sky images [4]: a) M101; b) NGC 290; c) M74; d) LH 95.

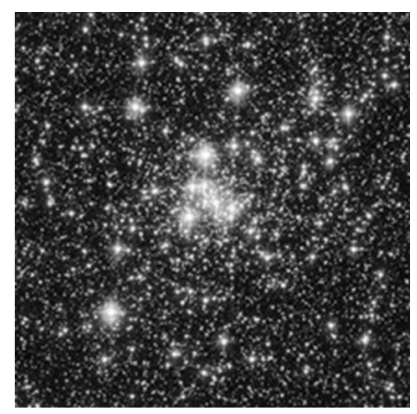

(a)

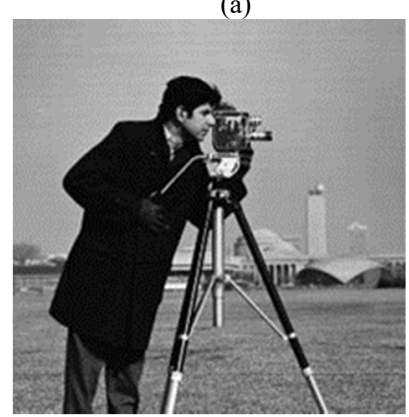

(c)

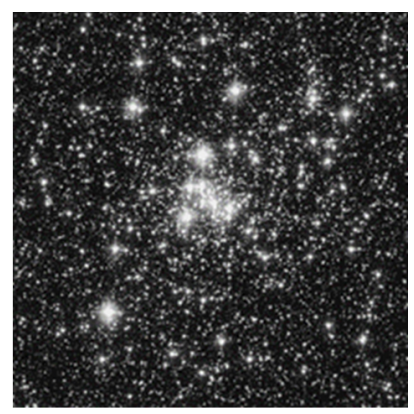

(b)

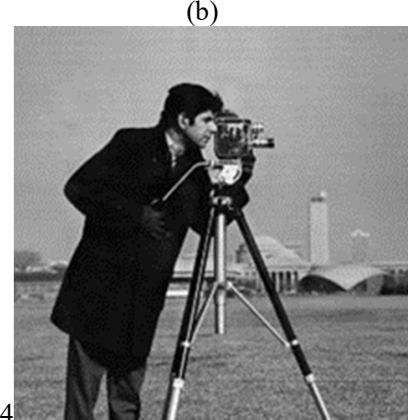

(d)
Fig. 2. Embedding \& Extraction for NGC 290: (a) NGC 290; (b) encoded (PSNR =37.6242); (c) watermark; (d) decoded.

TABLE I. DEEP SKY RESULTS

\begin{tabular}{|c|c|c|c|c|c|c|c|}
\hline Image & $\begin{array}{c}\text { DSCR } \\
\text { PSNR }\end{array}$ & $\begin{array}{c}\text { DSCR } \\
\text { MC }\end{array}$ & $\mathbf{d}$ & $\mathbf{t}$ & $\mathbf{s}$ & $\begin{array}{c}\text { DS } \\
\text { PSNR }\end{array}$ & $\begin{array}{c}\text { DS } \\
\text { MC }\end{array}$ \\
\hline M101 & $\mathbf{3 7 . 5 2 8 6}$ & 0.9614 & 9 & 0.1 & 1100 & 37.4955 & 0.9613 \\
\hline $\begin{array}{c}\text { NGC } \\
290\end{array}$ & $\mathbf{3 7 . 6 2 4 2}$ & $\mathbf{0 . 8 5 1 1}$ & 9 & 2.0 & 1111 & 37.4955 & 0.7988 \\
\hline M74 & $\mathbf{3 7 . 5 7 6 9}$ & 0.9219 & 8 & 0.7 & 1110 & 37.4956 & 0.9219 \\
\hline LH 95 & $\mathbf{3 7 . 5 9 9 6}$ & $\mathbf{0 . 8 8 9 0}$ & 8 & 0.1 & 1111 & 37.4955 & 0.8640 \\
\hline
\end{tabular}

Note: DS: Original DWT-SVD; DSCR: DWT-SVD with Curve-Fitting and Robust Regression; MC: Mean correlation; $d$ : Degree of the DSCR polynomial-fitting; $t$ : Tuning constant for DSCR robust regression; s: Binary string of DSCR (2.D.1).

Hence, we have an improvement of DWT-SVD via curvefitting and robust regression for each astronomy image. The most striking result is obtained on NGC 290, where we have an obvious advance especially in terms of robustness: a boosted mean correlation value of 0.8511 .

\section{3) Classical Images}

From the following results, one can easily see that, the proposed technique is not a specialized- for-deep-sky strategy. In this section we demonstrate DSCR embedding on well-known image processing data (https://homepages.cae.wisc.edu/ ece533/images/).

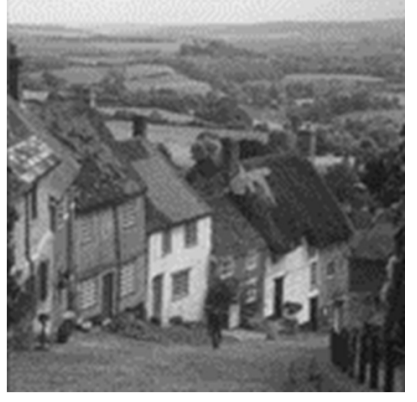

(a)

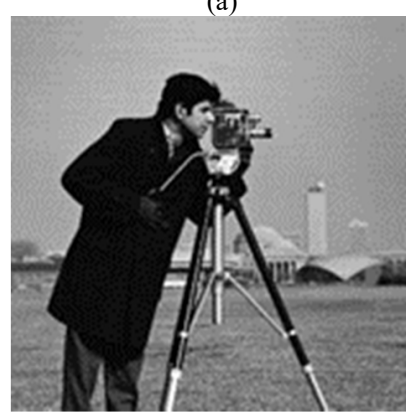

(c)

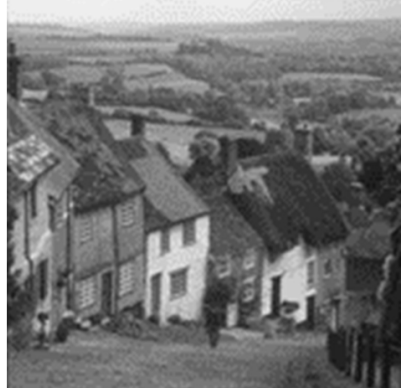

(b)

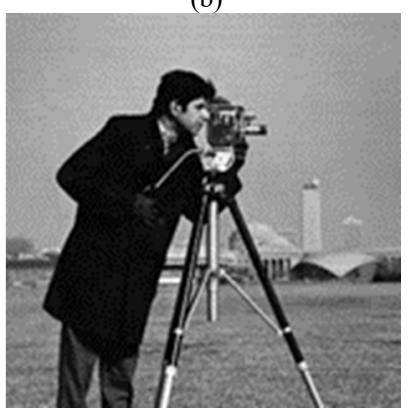

(d)
Fig. 3. Embedding \& extraction for Goldhill: (a) goldhill; (b) encoded (PSNR = 37.6144); (c) watermark; (d) decoded.

For Goldhill, the original, encoded and decoded images can be seen in Fig. 4. Detailed results for Airplane, Baboon, Boat, Goldhill, Barbara, Lenna and Watch are shown in the Table II.

TABLE II. RESULTS.

\begin{tabular}{|c|c|c|c|c|c|c|c|}
\hline Image & $\begin{array}{c}\text { DSCR } \\
\text { PSNR }\end{array}$ & $\begin{array}{c}\text { DSCR } \\
\text { MC }\end{array}$ & $\mathbf{d}$ & $\mathbf{t}$ & $\mathbf{s}$ & DS PSNR & $\begin{array}{c}\text { DS } \\
\text { MC }\end{array}$ \\
\hline Airplane & $\mathbf{3 7 . 6 1 0 1}$ & $\mathbf{0 . 8 5 8 9}$ & 9 & 0.1 & 1111 & 37.4955 & 0.8129 \\
\hline Baboon & $\mathbf{3 7 . 6 2 3 4}$ & $\mathbf{0 . 8 0 3 5}$ & 9 & 0.1 & 1111 & 37.4955 & 0.7621 \\
\hline Boat & $\mathbf{3 7 . 6 2 2 1}$ & $\mathbf{0 . 8 3 3 1}$ & 9 & 0.4 & 1111 & 37.4955 & 0.7930 \\
\hline Goldhill & $\mathbf{3 7 . 6 2 4 4}$ & $\mathbf{0 . 8 9 3 2}$ & 8 & 0.1 & 1111 & 37.4956 & 0.8331 \\
\hline Barbara & $\mathbf{3 7 . 6 2 1 7}$ & $\mathbf{0 . 8 2 1 0}$ & 8 & 0.4 & 1111 & 37.4955 & 0.8050 \\
\hline Lenna & $\mathbf{3 7 . 6 2 3 0}$ & $\mathbf{0 . 8 8 0 1}$ & 9 & 3.5 & 1111 & 37.4956 & 0.8712 \\
\hline Watch & $\mathbf{3 7 . 6 2 2 9}$ & $\mathbf{0 . 8 8 1 5}$ & 9 & 1.7 & 1111 & 37.4955 & 0.8550 \\
\hline
\end{tabular}

\section{CONCLUSIONS}

In this work, an idea based on expressing the set of watermark singular values by functions of the cover image's DWT domain singular values is implemented. Since such an approximation enforces a more consistent transform on the cover image's DWT singular values, a higher PSNR value is obtained. Moreover, together with the utilization of a twolevel robust regression, a boosting of the mean correlation value is achieved. Obviously, one can find more sophisticated ways of fusing the components, e.g. through Support Vector Regression, albeit the thematic concentration on iterative reweighted least-squares of this 
scheme.

\section{REFERENCES}

[1] E. Ganic, A. M. Eskicioglu, "Robust embedding of visual watermarks using discrete wavelet transform and singular value decomposition", Journal of Electronic Imaging, vol. 14, 2005.

[2] T. Peining, A. M. Eskicioglu, "A robust multiple watermarking scheme in the discrete wavelet transform domain", Internet Multimedia Management Systems, vol. 5, pp. 133-144, 2004.

[3] P. W. Holland, R. E. Welsch, "Robust regression using iter- atively reweighted least-squares", Communications in Statistics-Theory and Methods, vol. 6, no. 9, pp. 813-827, 1977. [Online]. Available: http://dx.doi.org/10.1080/03610927708827533

[4] Hubble webpage. [Online]. Available: http://hubblesite.org

[5] I. J. Cox, S. Member, J. Kilian, F. T. Leighton, T. Shamoon, "Secure spread spectrum watermarking for multimedia", Image Processing, IEEE Trans., vol. 6, no. 12, pp. 1673-1687, 1997. [Online] Available: http://dx.doi.org/10.1109/83.650120

[6] R. Dugad, K. Ratakonda, N. Ahuja, "A new wavelet-based scheme for watermarking images", in Proc. 1998 Int. Conf. Image Process. ICIP98, vol. 2, pp. 1-5, 1998. [Online]. Available: http://dx.doi.org/10.1109/icip.1998.723406

[7] G. Emir, A. M. Eskicioglu, "Robust DWT-SVD domain image watermarking: embedding data in all frequencies", in Proc. the 2004 Workshop on Multimedia and Security, 2004, pp. 166-174.

[8] E. Elbasi, A. M. Eskicioglu, I. Science, "A DWT-based robust semiblind image watermarking algorithm using two bands", Electronic Imaging, 2006. [Online]. Available: http://dx.doi.org/10.1117/ 12.651154
[9] O. Jane, E. Elbasi, "A new approach of nonblind watermarking methods based on DWT and SVD via LU decomposition", Turkish Journal of Electical. Engineering and Computer Sciences, vol. 22, no. 5, pp. 1354-1366, 2014. [Online]. Available: http://dx.doi.org/ 10.3906/elk-1212-75

[10] O. Jane, H. Gokhan, E. Elbasi, "A secure and robust watermarking algorithm based on the combination of DWT, SVD, and LU decomposition with Arnold 's cat map approach", in $8^{\text {th }}$ Int. Conf. on Electrical and Electronics Engineering, no. 3, pp. 306-310, 2014 [Online]. Available: http://dx.doi.org/10.1016/j.simpat.2009.09.004

[11] H.-C. Huang, W.-C. Fang, "Metadata-based image watermarking for copyright protection", Simul. Model. Pract. Theory, vol. 18, no. 4, pp. 436-445, 2010.

[12] M. Nasrin, Bee Ee Khoo, "Robust blind image watermarking scheme based on redundant discrete wavelet transform and singular value decomposition", AEU-Int. Journal of Electronics and Communications, vol. 6, no. 2, pp. 102-112, 2013.

[13] M. Anurag, et al., "Optimized gray-scale image watermarking using DWT-SVD and Firefly Algorithm", Expert Systems with Applications vol. 41, vol. 17, pp. 7858-7867, 2014.

[14] R. B. Wolfgang, E. J. Delp, R. B. Wolfgang, E. J. Delp, "Fragile watermarking using the VW2D watermark", in Proc. SPIE/IS\&T Int Conf. Secur. Watermarking Multimed. Contents, vol. 3657, 1999, pp. 204-213.

[15] A. Musrrat, Chang Wook Ahn, M. Pant, "A robust image watermarking technique using SVD and differential evolution in DCT domain", Optik-International Journal for Light and Electron Optics vol. 125, no. 1, pp. 428-434, 2014. [Online]. Available: http://dx.doi.org/10.1016/j.ijleo.2013.06.082. 